\title{
Correlated Quantitative Studies of the Neostriatum, Nucleus Accumbens, Substantia Nigra, and Ventral Tegmental Area in Normal and Weaver Mutant Mice
}

\author{
Shirley A. Bayer, ${ }^{1}$ Lazaros C. Triarhou, ${ }^{3}$ James D. Thomas, ${ }^{2}$ and Bernardino Ghetti ${ }^{3}$ \\ Departments of ${ }^{1}$ Biology and ${ }^{2}$ Psychology, Indiana Purdue University, and ${ }^{3}$ Department of Pathology and Laboratory \\ Medicine, Indiana University School of Medicine, Indianapolis, Indiana 46202
}

\begin{abstract}
Normal mice $(+/+)$ and homozygous weaver mutant mice ( $w v / w v$ ) at 1 year of age were used for three-dimensional computer-aided reconstructions of the nucleus accumbens (NA) and neostriatum (ST) and for quantitative estimations of the total number of medium-sized neurons in the NA and ST, and for the total number of tyrosine hydroxylase (TH)containing neurons in the ventral tegmental area (VTA) and substantia nigra (SN). The three-dimensional reconstructions showed that the weaver NA and ST are smaller than they are in $+/+$. Quantitative volumetric measurements of the NA and ST showed $w v / w v$ were smaller than $+/+$ by nonsignificant differences of $14 \%$ and $13 \%$, respectively. The $w v / w v$ group showed statistically significant depletion of neurons in all four structures. On average, NA neurons are reduced by $27 \%$, ST neurons by $22 \%$, VTA-TH neurons by $40 \%$, and SN-TH neurons by $79 \%$. In wv/wvanimals, there was a high positive correlation $(r=0.836)$ between the numbers of SN-TH neurons and ST neurons and a moderate positive correlation ( $r=0.534$ ) between the numbers of $\mathrm{SN}$ VTA neurons and NA neurons. The nuclei in TH-containing neurons in $w v / w v$ and $+/+$ had the same diameters, but in all animals, the $\mathrm{SN}-\mathrm{TH}$ neurons contained larger nuclei than the VTA-TH neurons. Cytoarchitectonic measurements in control and weaver NA and ST were also similar. In all animals, the NA contains more densely packed neurons with smaller nuclei than those in the ST.

[Key words: striatum, nucleus accumbens, ventral tegmental area, substantia nigra, weaver mutant mouse, cell death, volumetric cell counts, three-dimensional computer reconstructions]
\end{abstract}

Adult homozygous weaver ( $w v / w v)$ mutant mice have a severe depletion of dopamine neurons in the substantia nigra (Schmidt et al., 1982; Triarhou et al., 1986, 1988a; Gupta et al., 1987; Ghetti and Triarhou, 1992), and to a lesser extent in the ventral tegmental area (Triarhou et al., 1988a; Graybiel et al., 1990).

\footnotetext{
Received Nov. 11, 1993; revised May 20, 1994; accepted May 26, 1994.

We are grateful for the technical assistance of Kathy Wills, Daniele Drake, Mark Grosfeld, Paula Kong, Li Yuan, Eloise Turner, Lillian Fitzpatrick, and Julie Henderson. We also thank J. Altman for providing the photographic facilities in his laboratory and J. Simon for his helpful comments. This research was supported by NIH Grant NS27613.

Correspondence should be addressed to Shirley A. Bayer, Department of Biology, Indiana-Purdue University, 723 West Michigan Street, Indianapolis, IN $46205-5132$.
}

Copyright (C) 1994 Society for Neuroscience $0270-6474 / 94 / 146901-10 \$ 05.00 / 0$
Substantia nigra neurons project massively to the neostriatum (Rosegay, 1944; Ungerstedt, 1971; Nauta et al., 1974; Schwab et al., 1977; Faull and Mehler, 1978; Royce, 1978; Veening et al., 1980; Gerfen et al., 1982; Fisher et al., 1983; JimenezCastellanos and Graybiel, 1987), and the loss of nigral neurons is correlated with reduced dopamine content in the neostriatum of $w v / w v$ mutants (Schmidt et al., 1981, 1982). Ventral tegmental area neurons project massively to the nucleus accumbens (Ungerstedt, 1971; Carter and Fibiger, 1977; Nauta et al., 1978; Beckstead et al., 1979; Chronister et al., 1980; Groenewegen et al., 1980; Hökfelt et al., 1980; Newman and Winans, 1980; Kalivas and Miller, 1984; Gerfen et al., 1987a; Kalsbeek et al., 1987), and because of the milder cell loss in $w v / w v$ mutants, dopamine content is unchanged in the nucleus accumbens (Roffler-Tarlov and Graybiel, 1987). However, the nucleus accumbens in $w v / w v$ mutants has below-normal dopamine uptake and storage (Doucet et al., 1989; Roffler-Tarlov et al., 1990), and reduced dopamine D2 receptors (Kaseda et al., 1990). Dopaminergic neuronal degeneration has been seen as early as postnatal day 1 (P1) (Ghetti and Triarhou, 1992c) and on P16 and P20 (Ghetti and Triarhou, 1992a,b). Cell loss has been quantified in the $w v / w v$ substantia nigra on P20 (Triarhou et al., 1988a). The ventral tegmental area in young adults contains reduced immunostaining for tyrosine hydroxylase (Triarhou et al., 1988a; Graybiel et al., 1990) and a depletion of tyrosine hydroxylase-stained neurons (Triarhou et al., 1988a). In 1-yearold $w v / w v$ mice, the neostriatum has been deprived of normal dopamine levels for at least 11 months, and dopamine metabolism in the nucleus accumbens may have been abnormal for that same period of time.

The major focus of this article is to study quantitatively the neostriatum, nucleus accumbens, substantia nigra, and ventral tegmental area in 1-year-old control $(+/+)$ and $w v / w v$ mice to determine if there are any differences between them and if those differences can be correlated between structures. We address three questions. First, does the gross appearance of the striatum and nucleus accumbens (as determined by computer-assisted three-dimensional reconstructions) differ between $+/+$ and $w v /$ $w v$ mice? The three-dimensional images we show here indicate that the $w v / w v$ striatum and nucleus accumbens are slightly smaller than they are in $+/ t$, but they are very similar to $+/$ + in overall appearance. Second, does the total number of neurons in the neostriatum and nucleus accumbens differ between $+/+$ and $w v / w v$ ? We report here that $w v / w v$ animals have significantly fewer neurons than $+/+$ animals in both structures. Third, does the loss of dopamine neurons in the substantia nigra 
Table 1. The data of each control $(+/+)$ and each $w v / w v$ animal along with their group means $(\Sigma)$ and SD for the total numbers of neurons and the structural volumes of the nucleus accumbens (NA) and neostriatum (ST) and for the total numbers of TH-stained neurons in the ventral tegmental area (VTA) and substantia nigra (SN)

\begin{tabular}{|c|c|c|c|c|c|c|c|}
\hline \multirow[b]{3}{*}{$\begin{array}{l}\text { Animal } \\
\text { number }\end{array}$} & \multirow[b]{3}{*}{ Genotype } & \multirow{3}{*}{$\begin{array}{l}\text { NA } \\
\text { Total } \\
\text { number }\end{array}$} & \multirow{3}{*}{$\begin{array}{l}\text { Total } \\
\text { volume } \\
\left(\mathrm{mm}^{3}\right)\end{array}$} & \multicolumn{2}{|l|}{ ST } & \multirow{3}{*}{$\begin{array}{l}\text { VTA } \\
\text { Total } \\
\text { number }\end{array}$} & \multirow{3}{*}{$\begin{array}{l}\text { SN } \\
\text { Total } \\
\text { number }\end{array}$} \\
\hline & & & & \multirow[b]{2}{*}{$\begin{array}{l}\text { Total } \\
\text { number }\end{array}$} & \multirow{2}{*}{$\begin{array}{l}\text { Total } \\
\text { volume } \\
\left(\mathrm{mm}^{3}\right)\end{array}$} & & \\
\hline & & & & & & & \\
\hline $6196-1-1$ & $+/ t$ & 591,403 & 1.14 & $2,623,608$ & 8.89 & 3,216 & 4,137 \\
\hline $6196-1-2$ & $+/+$ & 589,625 & 1.00 & $2,950,618$ & 8.20 & 3,231 & 4,574 \\
\hline $1100-708-1$ & $+/ t$ & 669,895 & 1.06 & $2,913,513$ & 7.72 & 3,333 & 5,074 \\
\hline $1100-708-2$ & $+/ t$ & 579,146 & 0.96 & $3,113,314$ & 7.59 & 4,107 & 4,295 \\
\hline$\Sigma$ & & 607,517 & 1.04 & $2,900,263$ & 8.10 & 3,472 & 4,520 \\
\hline $\mathrm{SD}$ & & 41,935 & 0.08 & 203,829 & 0.59 & 427 & 411 \\
\hline $0700-118$ & $w v / w v$ & 372,435 & 1.05 & $1,852,022$ & 8.14 & 1,855 & 647 \\
\hline $0700-239$ & $w v / w v$ & 455,812 & 0.90 & $2,258,685$ & 6.90 & 1,711 & 974 \\
\hline $0700-242$ & $w v / w v$ & 476,098 & 0.86 & $2,609,937$ & 7.37 & 2,317 & 1,004 \\
\hline $0700-246$ & $w v / w v$ & 464,227 & 0.75 & $2,357,371$ & 5.87 & 2,440 & 1,115 \\
\hline 2 & & 442,143 & 0.89 & $2,269,504$ & 7.07 & 2,081 & 935 \\
\hline SD & & 47,211 & 0.12 & 315,184 & 0.95 & 352 & 201 \\
\hline
\end{tabular}

The $w v / w v$ means are significantly below $(p<0.05)$ the $+/+$ means for each cell number estimation.

and ventral tegmental area in $w v / w v$ correlate with any neuron loss in the neostriatum and nucleus accumbens? The counts of tyrosine hydroxylase-stained neurons show severe reduction in the substantia nigra and, by the age of 1 year, substantial reduction in the ventral tegmental area. In $w v / w v$, there is a high positive correlation between cell losses in the substantia nigra and the neostriatum, and a moderate positive correlation between cell losses in the ventral tegmental area and the nucleus accumbens.

\section{Materials and Methods}

For the three-dimensional reconstruction study and for all quantitative cell number estimation studies, the animal subjects were obtained from the colony of control and $w v / w v$ mice at Indiana University School of Medicine maintained on the B6CBA- $4^{\mathrm{w}-\mathrm{J}} / A$ hybrid stock. All animals were killed on their first birthday by overdosing with sodium pentobarbital $(35 \mathrm{mg} / \mathrm{kg})$ and perfusion through the heart with $10 \%$ neutral buffered formalin. All the $w v / w v$ animals showed the abnormal "weaving" gait patterns. In addition, the cerebellum was sectioned to confirm the $w v / w v$ phenotype. In $w v / w v$, the cerebellum is severely reduced in size and has very few granule cells (Rakic and Sidman, 1973). For the cell estimation studies, the animals were also perfused with Bouin's fixative to enhance the immunocytochemical staining of tyrosine hydroxylase-containing neurons following the protocol of Triarhou et al (1988a). The methods chosen for three-dimensional reconstruction of the neostriatum and nucleus accumbens were identical to those used by Bayer and Altman (1991) in their study of neocortical development. Although both $+/+$ and $w v / w v$ have sparsely scattered large neurons in the neostriatum and clusters of small neurons in the nucleus accumbens, we quantified only the medium-sized neurons in each structure, which make up the major part of the total population. The methods chosen for estimation of the medium-sized neurons were slightly modified from those used by Bayer et al. (1982) and Bayer (1982) in their study of the dentate granule cell population. That method is especially suitable for estimating cell numbers in large neuronal populations in structures with well-defined boundaries. The method is not based on cell counts but rather on the proportional volume taken up by neuronal nuclei in the entire structure. Since both the substantia nigra and ventral tegmental area do not have well-defined borders with adjacent brain structures, we used a different estimation procedure based on cell counts. The raw cell counts were corrected for split nuclei using the equation of Abercrombie (1946) as modified by Konigsmark (1970). The average nuclear diameter was determined using the iterative subtraction method of Hen- dry (1976). The several steps involved in the three-dimensional reconstruction process and the cell number estimation studies are described in detail in the Appendix.

\section{Results}

Three-dimensional reconstructions of the nucleus accumbens and the neostriatum

Figure 1 shows the computer-reconstructed nucleus accumbens (yellow) and neostriatum (green) with the same orientation and position as they are on the right side of the brain in both a control animal ( $+/+$; left) and homozygous weaver ( $w v / w v$; right) from three different views (Fig. $1 A-C$ ) at exactly the same magnification (scale bars in $C$ ). When these two individual animals are compared, the weaver neostriatum and nucleus accumbens is smaller (by 25\%) than the same structures in the control. However, the overall shape and orientation of these brain structures is virtually identical in the two genotypes. The smaller nucleus accumbens lies ventromedial and anterior, while the much larger neostriatum lies dorsal, lateral and posterior. The prominent depression in the posteromedial neostriatum (Fig. $1 C$ ) is caused by enlargement of the thalamus and the hippocampus. At its most posterior part, only a thin wafer of neostriatum is wedged between the neocortex on the exterior and the hippocampus and thalamus on the interior. The surrounding brain structures were not reconstructed, because their images would have masked the neostriatum and nucleus accumbens.

\section{Quantitative differences between control and weaver mice in the accumbens-neostriatal complex}

The nucleus accumbens. The total number of medium-sized neurons in the $w v / w v$ nucleus accumbens is reduced by approximately $27 \%$ from the $+/+$ total mean number (from 607,517 to 442,143; Table 1). These differences are significant using a single-factor analysis of variance (ANOVA) $[F(1,6)=27.43, p$ $<0.001]$. The total volume of the $w v / w v$ nucleus accumbens is reduced $14 \%$ below the $+/+$ volume (from 1.04 to $0.89 \mathrm{~mm}^{3}$; Table 1). Even though the data on total volume show the same trend as those on total number of cells, the ANOVA indicates 
they are not significantly different $[F(1,6)=4.18, p>0.05]$, mainly due to the larger variability in the $w v / w v$ group ( $w v / w v$ $\mathrm{SD}$ is 1.5 times greater than the $+/+\mathrm{SD}$; see Table 1).

The neostriatum. The total number of medium-sized neurons in the $w v / w v$ neostriatum is reduced by approximately $22 \%$ from the $+/+$ total mean number (from 2,900,263 to 2,269,504; Table 1). The ANOVA indicates that these differences are significant $[F(1,6)=11.3, p=0.02]$. The total volume of the $w v /$ $w v$ neostriatum is reduced $13 \%$ below the $+/+$ volume (from 8.1 to $7.07 \mathrm{~mm}^{3}$; Table 1). Even though the data on total volume show the same trend as those on total number of cells, the ANOVA indicates they are not significantly different $[F(1,6)=$ $3.4, p>0.05$ ], mainly due to the larger variability in the $w v /$ $w v$ group ( $w v / w v \mathrm{SD}$ is 1.6 times greater than the $+/+\mathrm{SD}$; see Table 1).

\section{Quantitative differences between control and weaver mice in the ventral tegmental area and the substantia nigra}

The substantial reductions in the ventral tegmental area (VTA) and the substantia nigra (SN) between $+/+$ and $w v / w v$ are easily observed without any quantitative measurement (Fig. $2 A, B$ ). The total numbers of tyrosine hydroxylase ( $\mathrm{TH}$ )-containing neurons in the $w v / w v$ VTA is $40 \%$ below the $+1+$ total numbers (from 3472 to 2081; Table 1). These differences are significant using a two-sample $t$ test $[t=5.03, p=0.002$ (one tailed)]. The total numbers of TH neurons in the $w v / w v \mathrm{SN}$ is $79 \%$ below the $+1+$ total numbers (from 4520 to 935 , Table 1 ). These differences are significant using a two-sample $t$ test $[t=15.66, p=$ 0.00005 (one tailed)].

\section{Correlations between cell loss in the ventral tegmental area and substantia nigra and cell loss in the nucleus accumbens and the neostriatum}

Table 1 lists the number and genotype of each animal for six different measures in the four structures that were analyzed. The $x-y$ scattergrams in Figure 3 show completely nonoverlapping data clusters for the $+1+$ (squares) and $w v / w v$ (circles) when the total numbers of neurons in the four structures analyzed are compared (Table 1, columns 3, 5, 7, 8). When Pearson correlation coefficients are determined for the combined groups, there are strong positive relationships between the total number of neurons in the ventral tegmental area and the nucleus accumbens ( $r=0.829$; Fig. 3, left), and between the substantia nigra and the neostriatum ( $r=0.839$; Fig. 3, right) because $w v / w v$ always have fewer neurons than control animals. The disparity between groups is largely responsible for these high correlations. The correlation coefficient of the control group alone drops dramatically in both comparisons (VTA to NA, $r=-0.34$; SN to ST, $r=0.271$ ), indicating virtually no relationship between the total numbers of VTA and NA neurons on the one hand and the total numbers of SN and ST neurons on the other. However, the correlation coefficient for the wcaver group alone is much higher in both comparisons (VTA to NA, $r=0.534$; SN to ST, $r=0.836$ ), indicating that neuronal loss in the midbrain may be linked to neuronal loss in the accumbens/neostriatal complex.

Cytoarchitectonic differences between the neostriatum, nucleus accumbens, ventral tegmental area, and substantia nigra

During the close observation of the four brain structures that were analyzed, consistent differences were noticed between them.
For example, tyrosine hydroxylase-stained neurons in the substantia nigra appeared to be larger than those in the ventral tegmental area (compare Fig. $2 C, D$ ). The data collected to determine average nuclear diameter indicated that nuclei in substantia nigra neurons were approximately $13 \%$ larger than those in ventral tegmental area neurons. The two-sample $t$ test indicated no differences between $+/+$ and $w v / w v$ regarding nuclear diameter in either structure (all $p>0.05$ ) so the data were combined for the statistical tests. Figure $4 A$ shows the data from individual animals (animals $1-4$ are $+/+; 6-8$ are $w v / w v$ ). In every animal there were smaller neurons in the ventral tegmental area than those in the substantia nigra. A within-subjects $t$ test indicated a highly significant statistical difference $[t(7)=-14.63$, $p<0.0001]$. On average, a nucleus in a VTA neuron had a diameter of $9.44 \mu \mathrm{m}$, while a nucleus in an SN neuron had a diameter of $10.7 \mu \mathrm{m}$.

With regard to the nucleus accumbens and neostriatum, it became easy to determine which structure was being sampled in the high-magnification photographic series because the nucleus accumbens contains closely packed neurons with smaller nuclei (Fig. 5A), while the neostriatum, except for a thin dorsomedial strip, has more widely spaced neurons with larger nuclei (Fig. $5 B$ ). Because the ANOVA indicated no significant differences between $+/+$ and $w v / w v$ in the average nuclear volume and nuclear packing density in both the nucleus accumbens and the neostriatum $[F(1,6)=0.23$ to 1.36 , all $p>0.05]$, the data were combined. Figure $4 B$ shows that the average nuclear volume in the nucleus accumbens is smaller in every animal than it is in the neostriatum (animals $1-4$ are $+/+; 5-8$ are $w v /$ $w v)$. The mean average nuclear volume in the neostriatum is $2.8 \times 10^{-7} \mathrm{~mm}^{3}$, which is nearly $23 \%$ larger than the mean average nuclear volume in the nucleus accumbens $2.28 \times 10^{-7}$ $\mathrm{mm}^{3}$. A within-subjects $t$ test indicated a highly significant statistical difference $[t(7)=-8.33, p<0.0001]$. Figure $4 C$ shows that nuclear packing density, as measured by the proportion of the sample areas occupied by nuclei, is always lower in the neostriatum than it is in the nucleus accumbens. On average, only $9.2 \%$ of a sample area in the neostriatum is taken up by medium-sized neuronal nuclei, while $11.33 \%$ of the sample areas in the nucleus accumbens are occupied by neuronal nuclei. A within subjects $t$ test indicated a highly significant difference $[t(7)=7.54, p<0.0001]$.

\section{Discussion}

\section{Gross anatomy of the neostriatum and nucleus accumbens}

To our knowledge, this is the first three-dimensional reconstruction of the neostriatum and nucleus accumbens that has been carried out. The reconstructions in Figure 1 show an overall size reduction in the $w v / w v$ but otherwise normal morphology is maintained in spite of abnormal dopamine innervation. This is not surprising in view of the fact that cursory light microscopic examination of conventionally stained histological sections of both the neostriatum and nucleus accumbens appear to be normal in $w v / w v$. It has also been reported that the distribution of $\mathrm{D}_{1}$ dopamine receptors is normal in the $w v / w v$ neostriatum (Ohta et al., 1989). However, motor function ascribed to dopaminergic innervation of the basal ganglia is abnormal in $w v / w v$ (Triarhou and Ghetti, 1987), and the volumetric studies show that there are significant depletions in the total number of basal ganglionic neurons. 

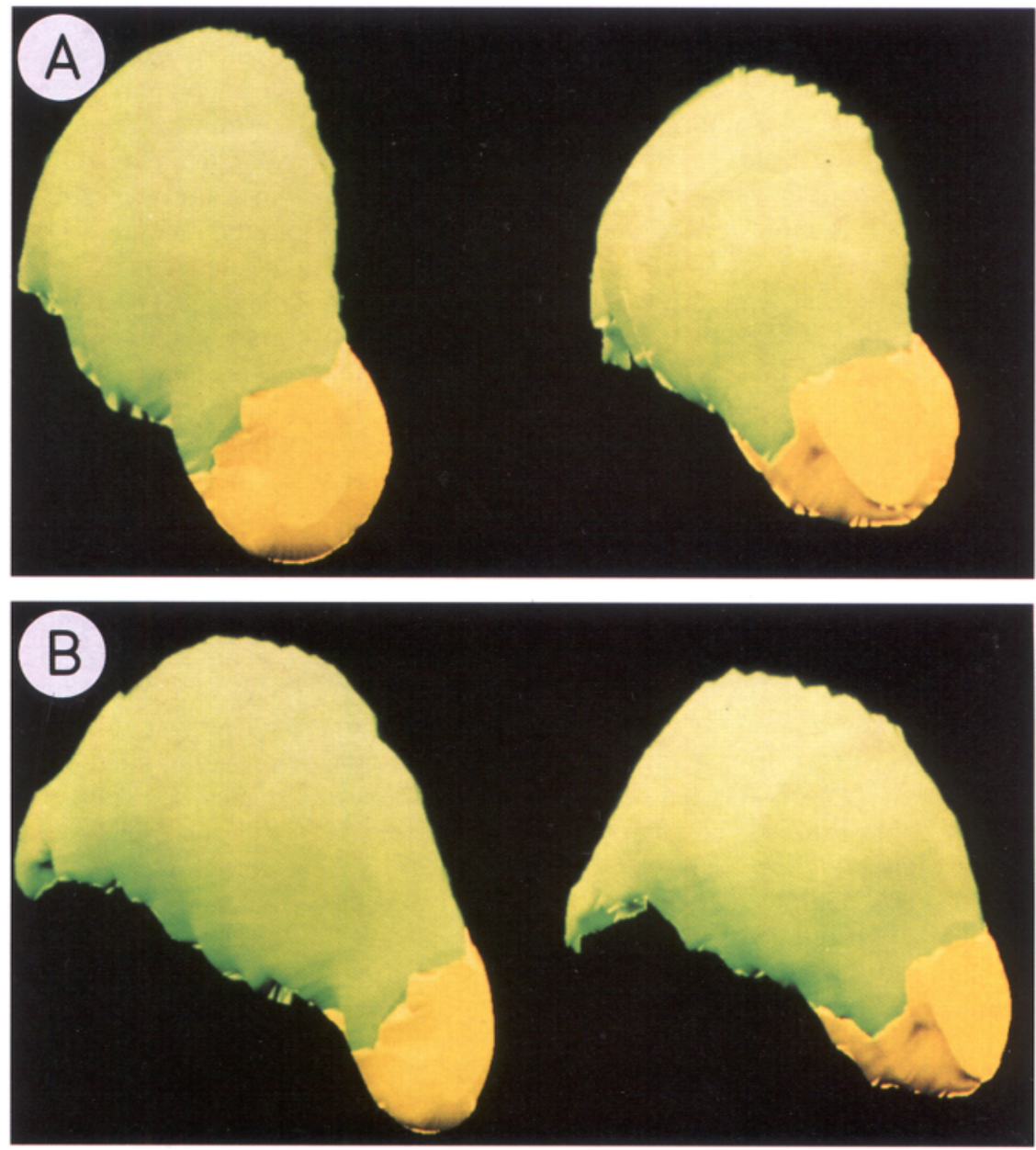

Figure 1. Three-dimensional reconstructions of the nucleus accumbens (yellow) and striatum (green) in $+/+$ (left) and homozygous $w v / w v(r i g h t) . A$, Front view (the observer is viewing straight down the z-axis, dorsal is on the top, ventral on the bottom, lateral is left, and medial right). $B$, Lateral view (the image in $A$ has been rotated $+45^{\circ}$ in the y-axis). $C$, Medial view (the image in $A$ has been rotated $-90^{\circ}$ in the $\mathrm{y}$-axis, anterior is left, and posterior is right). In this view, the $w v / w v$ accumbens/neostriatal complex appears larger than that in $+/+$ because it is shorter in the $\mathrm{z}$-axis and slightly broader in the anterior part of the $\mathrm{x}$-axis, which produces the optical illusion of more bulk. This view is shown to demonstrate essentially normal morphology in the medial $w v / w v$ accumbens/neostriatal complex.

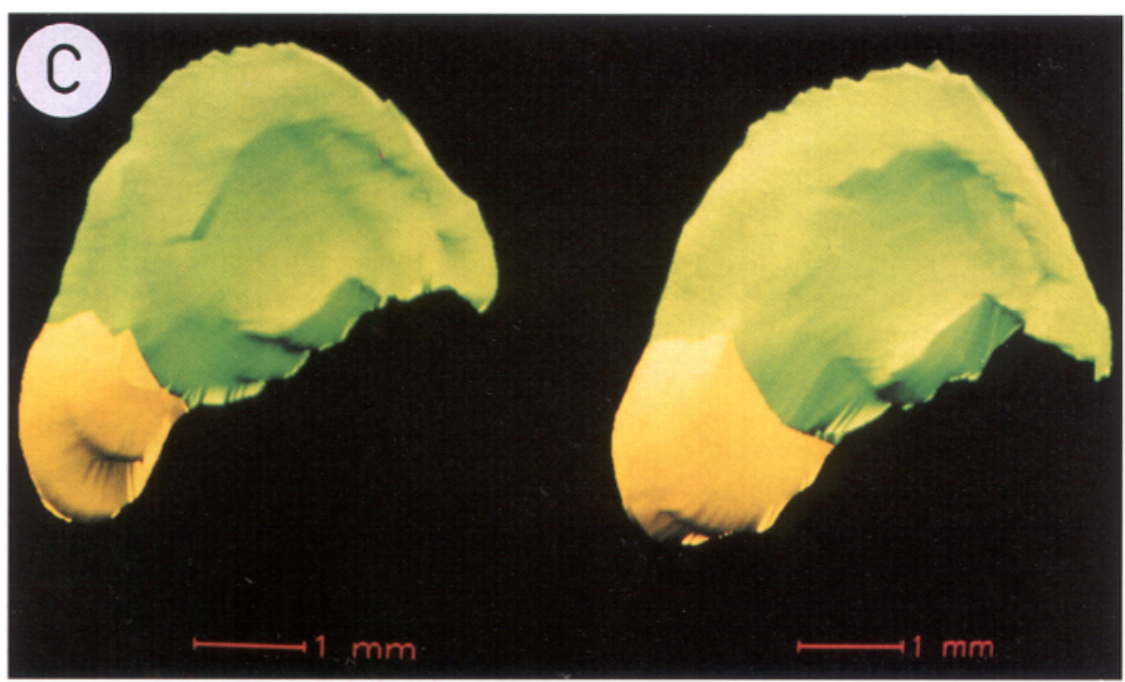

\section{Cell depletion in weaver mutants}

The data in Table 1 show severe depletion of substantia nigra (SN) neurons in 1-year-old $w v / w v(\sim 80 \%$ below $+/+)$ and substantial depletion in the ventral tegmental area (VTA; $40 \%$ below $+/+$ ). These are greater than the deficits reported by Triarhou et al. (1988a) in 90-d-old $w v / w v$ (SN, 69\%; VTA, 26\%) but are less than the $85 \%$ reduction in the $\mathrm{SN}$ found in 2-year-old $w v / w v$ (Ghetti and Triarhou, 1992). Taken together, these stud- ies establish that neuronal loss is progressive with age in both structures with the major wave occurring in the first $90 \mathrm{~d}$. Recently, Sauer and Oertel (1994) demonstrated that degenerative events in the midbrain dopaminergic cell bodies occur only after initial changes at the level of the axon terminals. Several studies have reported either anatomically or neurochemically abnormal dopamine terminals in the $w v / w v$ neostriatum and nucleus accumbens (Triarhou et al., 1988b; Doucet et al., 1989; Kaseda et al., 1990; Roffler-Tarlov et al., 1990), and the progressive 

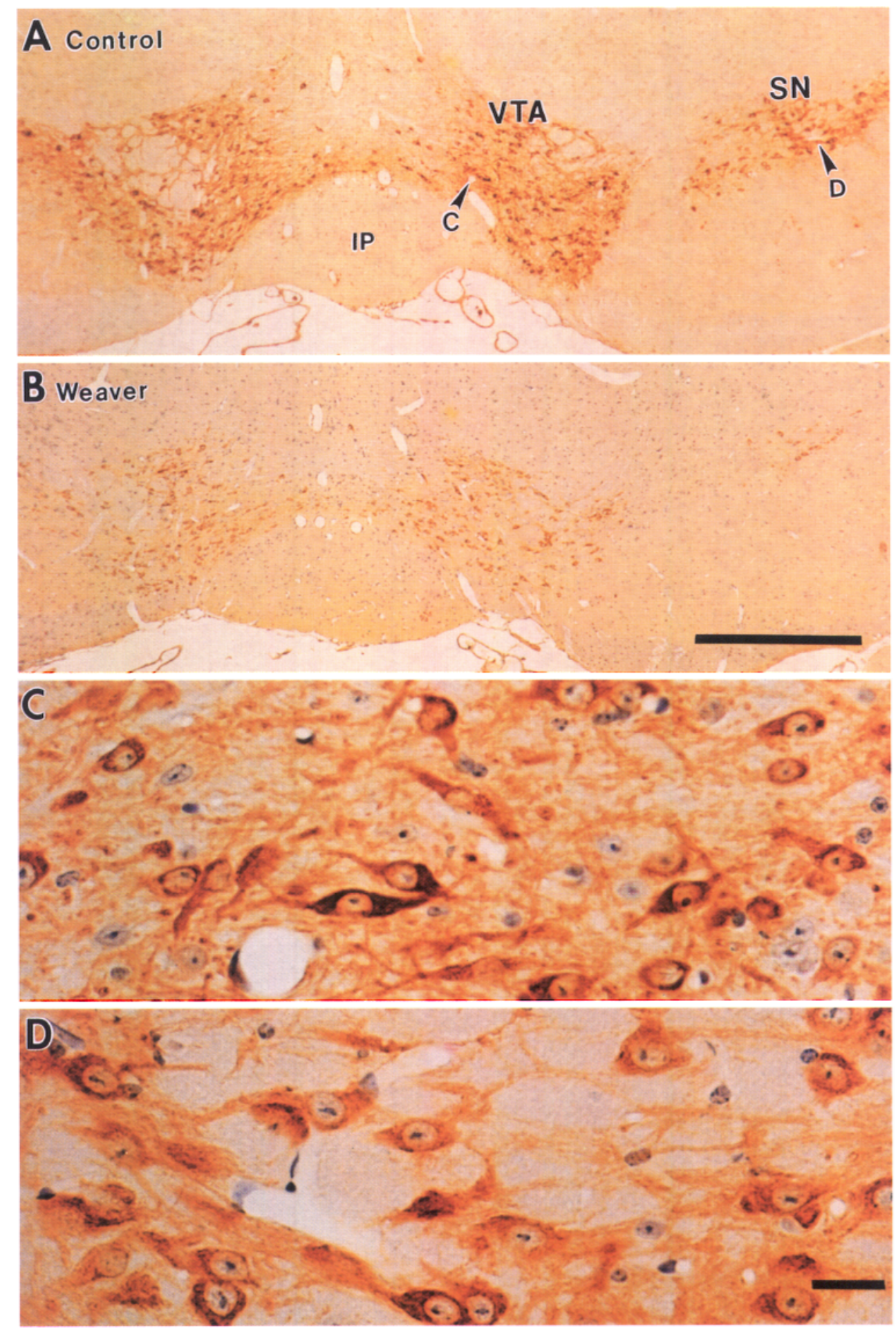

Figure 2. Illustrations of the dramatic depletion of tyrosine hydroxylase neurons in the midbrains of homozygous weavers $(B)$ compared to controls $(A)$ $(I P$, interpeduncular nucleus; $S N$, substantia nigra pars compacta; $V T A$, ventral tegmental area; $6 \mu \mathrm{m}$ paraffin sections counterstained with hematoxylin). The areas labeled $C$ and $D$ in $A$ are shown at higher magnification. $C$ shows the smaller neurons in the VTA, and $D$ shows the larger neurons in the $\mathrm{SN}$. Scale bars: $A$ and $B, 0.5 \mathrm{~mm} ; C$ and $D, 0.25$ $\mu \mathrm{m}$. decline in midbrain dopamine neurons may be related to initial deficits in their axon terminals.

The data presented in Table 1 indicate that the $w v / w v$ nucleus accumbens (NA) and neostriatum (ST) have $73 \%$ and $78 \%$, respectively, of the normal number of neurons. Although other studies have speculated on neuronal loss in the ST (Kaseda et al., 1990), and degeneration has actually been seen in the subependymal layer of the developing ST (Ghetti and Triarhou, 1992c), the present report is the first to demonstrate neuronal loss quantitatively in both NA and ST. Furthermore, there is no overlap between the data on total neuron number (Table 1, Fig. 3) from the control and weaver groups even though one homozygous weaver (animal 0700-118) has a larger ST and NA than some of the control animals.

A chief aim of this research is to see if loss of tyrosine hydroxylase neurons in the VTA/SN of $w v / w v$ correlates with neuronal loss in the NA/ST complex. There are at least three possible causes of neuronal loss in the NA and ST. First, the missing 
VTA:NA

- CONTROL - weAver

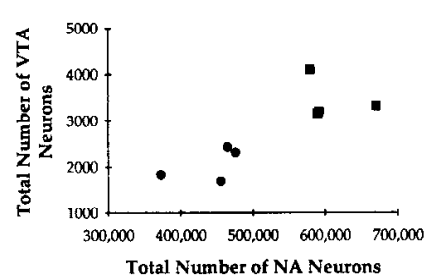

SN:ST

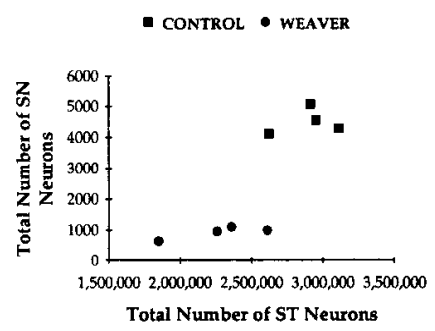

Figure 3. Scattergrams comparing the total numbers of neurons in anatomically related structures in controls (squares) and homozygous weaver mutants (circles). On the left, the ventral tegmental area (y-axis) is compared to the nucleus accumbens $(x$-axis). On the right, the substantia nigra (y-axis) is compared to the neostriatum (x-axis). In both comparisons, controls have more neurons than weavers. Within the weaver group, larger numbers of neurons in the NA or ST correlates with slightly more surviving tyrosine hydroxylase-stained neurons in the midbrain, while that relationship does not hold in the control group.

neurons may require normal dopamine levels and normal innervation for survival. From lesion studies in the olfactory system (and other brain systems), it is known that some neurons die after they are deprived of a major afferent input (Carlsen et al., 1982). It is also known that the few surviving dopamine axon terminals in the weaver neostriatum fail to establish an adequate synaptic connectivity pattern, thus contributing further to functional dopamine denervation (Triarhou et al., 1988b). Although only four animals in each group were used in this study, the positive correlation in $w v / w v$ between the total numbers of neurons in the VTA and the NA on the one hand (Fig. 3 , left), and the very high positive corrclation between total numbers of SN and ST neurons on the other (Fig. 3, right) support the hypothesis that some neurons in the NA and ST require dopaminergic input for survival. However, more animals would have to be analyzed to see if the positive correlations reported here are repeatable. This hypothesis is further supported by a recent quantitative study of multiple systems atrophy in humans where neuronal losses in the substantia nigra and the caudate putamen complex were correlated (Kume et al., 1993). A second possibility is that the weaver gene may have a direct lethal effect on a small proportion of accumbens and neostriatal neurons. The best way to test this possibility will be to estimate the total number of ST and NA neurons before the major period of cell death in the midbrain. Studies on dopamine content in preweaned $w v / w v$ (Roffler-Tarlov and Graybiel, 1987; Simon et al., 1994) suggest that the major deficit in dopamine occurs after postnatal days $7-10$. Thus, we assume that prior to P7, the numbers of SN and VTA neurons in $w v / w v$ may be closer to the control levels in spite of the evidence of degeneration at the time of birth (Ghetti and Triarhou, 1992c). We have collected a litter of obligatory homozygous $w v / w v$ (offspring of a mating between homozygous parents) on the day of birth and plan to investigate this possibility by quantifying the four structures at that age. A third possibility is that the weaver gene might cause a general depletion of neurons throughout the nervous system. To investigate that, volumetric studies of neuron

Figure 4. $A$, The average neuronal diameter in the ventral tegmental area (dark bars) and the substantia nigra (light hars) in the eight animals used in this study (animals $1-4$ are $+/ t ; 5-8$ are $w v / w v$ ). Note that in

$\mathbf{A}$

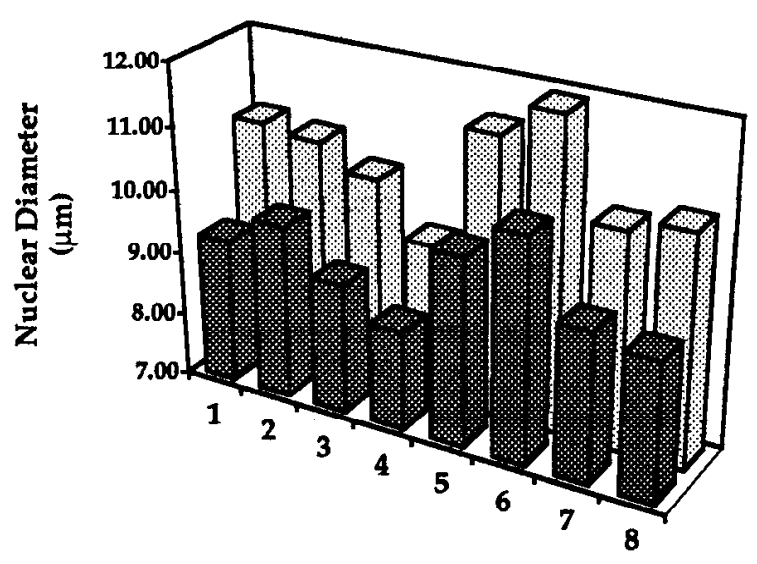

B

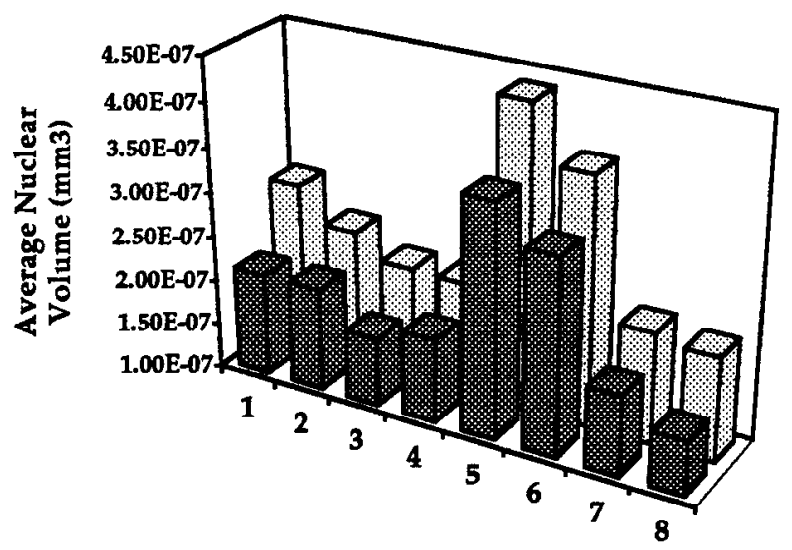

C 圈 ST NA

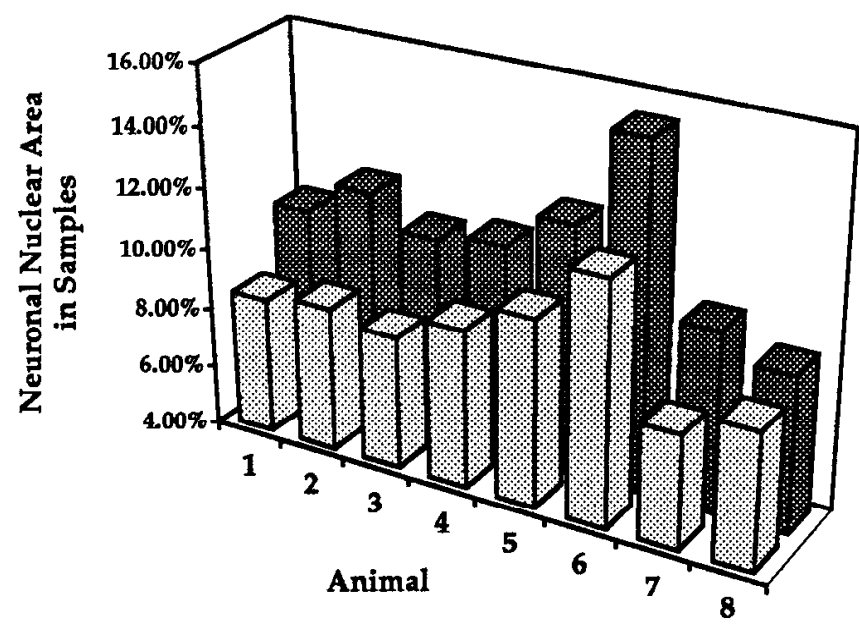

every animal, substantia nigra nuclei are larger than those in the ventral tegmental area. $B$, The average nuclear volume in the neostriatum ( $S T$; light bars) and the nucleus accumbens (NA; dark bars). Note that in every animal, neostriatal nuclei are larger than those in the nucleus accumbens. $C$, Nuclear packing density in the neostriatum (ST; light bars) and the nucleus accumbens (NA; dark bars). Note that in every animal, neostriatal nuclei are less densely packed than those in the nucleus accumbens. 

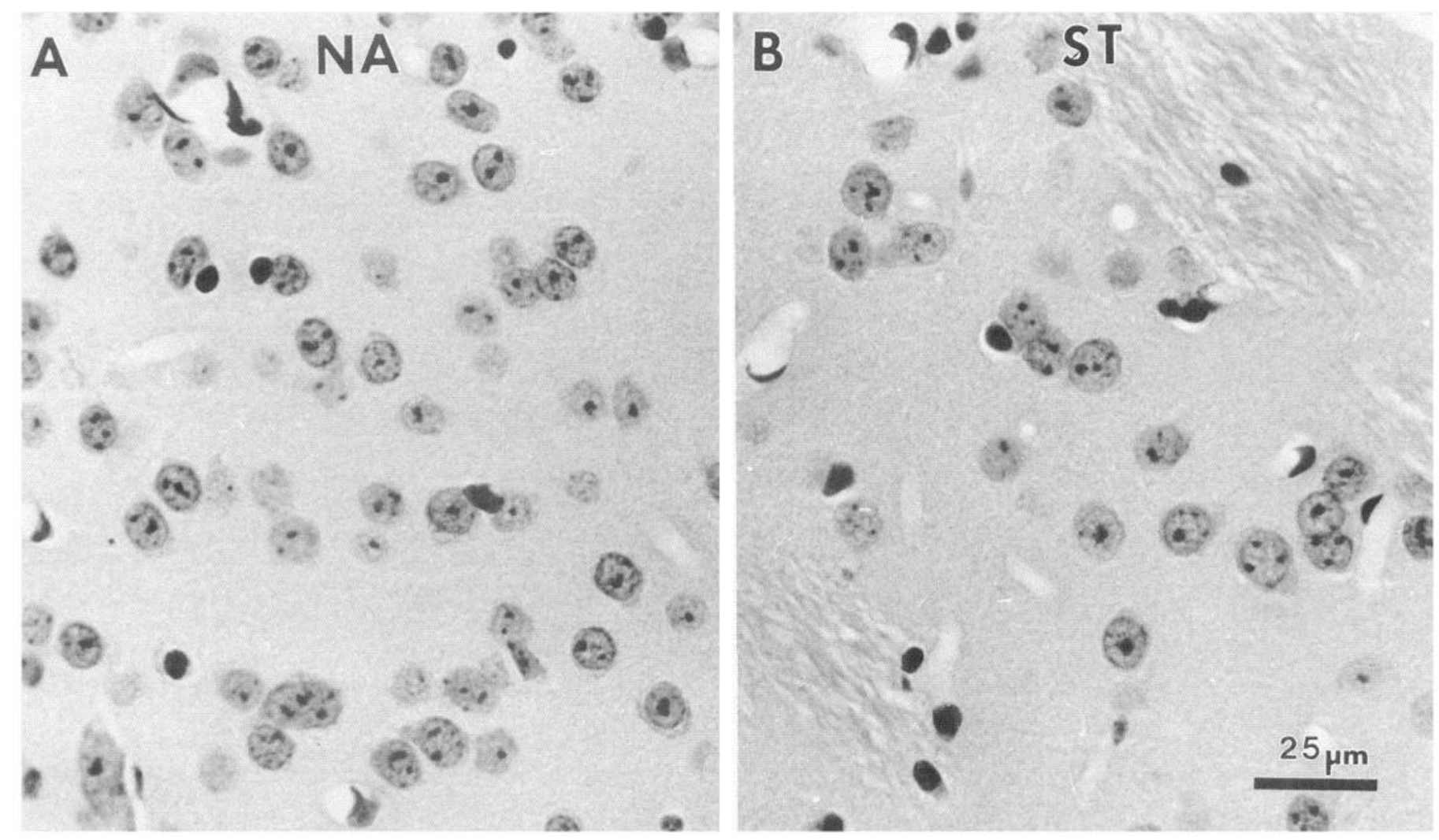

Figure 5. Randomly selected high-magnification samples of $(A)$ the nucleus accumbens $(N A)$ and $(B)$ the neostriatum $(S T)$ from control animal 6196-1-1. Typically, the NA has smaller nuclei that tend to be more closely packed than those in the ST (6 $\mu \mathrm{m}$ paraffin sections, hematoxylin stain).

numbers in a brain structure not receiving any dopamine innervation would have to be compared in $w v / w v$ and $+/+$ to see if neuronal numbers are similar in both groups. We plan to investigate the third possibility in future studies.

The data on cell loss in the ST can be related to neurochemical studies on dopamine receptor densities in the dorsolateral neostriatum (Kaseda et al., 1987, 1990; Ohta et al., 1989) that indicate an upregulation in homozygous $w v / w v$ at 6 months followed by a drop to below control levels by 12 months. Kaseda and coworkers found reduced protein content in the neostriatum of homozygous $w v / w v$ s, and hypothesized that cell loss may have occurred between 6 months and 1 year. The present study provides evidence for striatal cell loss, which may explain why dopamine $\mathrm{D}_{2}$ receptors are reduced to low levels at one year of age. It is not known if neuronal loss in the neostriatum is a progressive phenomenon. However, we have collected some 2 -year-old animals for a volumetric study similar to this one to see if there are greater depletions in ST and NA.

\section{Greater animal variability in wv/wv mutants}

The relatively large interindividual variances in $w v / w v$ recorded in Table 1 call for further comment. The $w v / w v$ mutation is maintained by repeated crossing of affected or carrier animals to a $\mathrm{C} 57 \mathrm{BL} / 6 \times \mathrm{CBA} \mathrm{F} 1$ hybrid mate, and intercrossing the progeny to generate litters segregating for $w v / w v$ homozygotes. These litters will also be segregating at all other genetic loci at which the C57BL/6 and CBA strains carry different alleles. This uncontrolled and undefined genetic variability is a likely factor in the variances, and implies that it might be possible to identify other genes that quantitatively modulate the dopaminergic system. Other possible reasons for the variance relate to interinvestigator variability (see Appendix) and undefined nongenetic variability among the mice.

\section{Cytoarchitectonic differences}

Statistical tests indicated that the data on cell diameter in the VTA and SN and the data on nuclear size and packing density in the NA and ST were not different between $+/+$ and $w v / w v$. Such a finding implies that the surviving neurons in the VTA and $\mathrm{SN}$ maintain a normal appearance even though the intensity of tyrosine hydroxylase staining was always lower in $w v / w v$ than in $+/+$ animals (compare Fig. $2 A, B$ ). The normal cytoarchitecture in the NA and the ST in $w v / w v$ is maintained in spite of severely depleted dopamine levels and abnormal dopamine connections.

However, there are clear cytoarchitcctonic differences between the VTA and the SN and between the NA and the ST. The data in Figure $4 A$ indicate that the nuclei of tyrosine hydroxylase-immunoreactive neurons in the $\mathrm{SN}$ are larger than those in the VTA. That agrees with the study of German and Manaye (1993) in rats where both the long and short axes of SN neurons were found to be larger than the same measures in VTA neurons. The data in Figure $4, B$ and $C$, indicate that the ST contains larger neurons that are less densely packed than those in the NA. No doubt, the decreased packing density in the ST is due to the thick bundles of axons coursing through it and connecting to various neocortical areas. To our knowledge, a quantitative study comparing nuclear size and packing density 
in these two structures has not been done. Mensah (1977) observed that the dorsolateral part of the neostriatum had larger neurons than medial or ventral parts. Our observations agree with that. In fact, dorsomedial parts of the neostriatum appear very similar to the nucleus accumbens. Herkenham (1984) did an extensive study of cell clustering in the nucleus accumbens of rats, observing that clusters are more prevalent near the ventromedial edge than in other parts. Our observations in mice indicate that clustering can be found throughout the nucleus, and clustering is probably more prominent in mice than it is in rats.

\section{Appendix}

Quantitative studies of the neostriatum and nucleus accumbens: methodological details

Three-dimensional reconstructions. For three-dimensional reconstructions, one representative control female $(+/+)$ and one representative homozygous weaver female $(w v / w v)$ were killed on their first birthday by perfusion through the heart with $10 \%$ neutral buffered formalin. The tissue block containing the neostriatum and nucleus accumbens on the right side of the brain was embedded in methacrylate (JB4, Polysciences) after dehydration. Plastic embedding was used because plastic sections have more regular expansion in the tissue bath after sectioning, which eliminates some of the artifacts that often show up in three-dimensional reconstructions of paraffin-sectioned material. Serial sections ( $3 \mu \mathrm{m}$, every 20 th) were placed on microscopic slides and were stained with toluidine blue.

The three-dimensional reconstruction method is described in detail elsewhere (Bayer and Altman, 1991). Briefly, low-magnification photographs of sections approximately $100 \mu \mathrm{m}$ apart were taken with a photomacroscope (Leitz/Wild) connected to an automatic exposure unit. The photographs were printed at a final magnification of $35 \times$, and the outlines of the neostriatum and nucleus accumbens were drawn on the photographs with a rapidograph pen.

To produce a three-dimensional reconstruction, it is necessary to place the sections in register, as they are in the brain before sectioning. Using a sagittally sectioned mouse brain from approximately the same age (taken from our histological set of normal specimens), we determined the curvature of the dorsal part of the neostriatum. Three fiducial marks were placed onto a the photograph of a section at a level midway between the anteroposterior extent of the striatum; this became the "orientation section." The orientation section was taped onto a light board for transillumination, and the photograph of the section in front of it was placed on top. Several anatomical landmarks were lined up along with the offset between the tops of the two sections (determined by calculating the distance between them and the curvature from the sagittal section photograph). The fiducial marks from the orientation section photo were copied onto the photo of the section being aligned. The orientation section was removed from the light board, and the fiducial marks around the section that had just been aligned were used as a template to align the next-most anterior section. This process was continued until all sections in front of the orientation section had been aligned. The same procedure was used to align the sections behind the orientation section.

The photographs were next placed onto a Summagraphics digitizer interfaced to an IBM computer (PS/2, model 60). The fiducial marks along with the contours around the neostriatum and the nucleus accumbens were traced by hand with the cross hair of a cursor. A program stored the $\mathrm{x}$ - and $\mathrm{y}$-coordinates of closely spaced points on the contours, and showed the outlines of the contours on the screen during the tracing. The section number was used as a code for the z-coordinate. The entire data file was transferred to a Silicon Graphics workstation (IRIS 4D20), where a conversion program (MOR2DAT) aligned the sections using the fiducial marks. That file was read by SKANDA, a three-dimensional reconstruction program written by Dr. J. Prothero and his coworkers at the University of Washington in Seattle. SKANDA constructed solid surfaces by drawing polygons between points on adjacent contours and filling them in with the selected color (green, neostriatum; yellow, nucleus accumbens; Fig. 1).

Determination of the total number of neurons. For this part of the study, four control females $(+/+)$ and four homozygous weaver females $(w v / w v)$ from our retired breeding stock were perfused through the heart, first with $10 \%$ neutral buffered formalin and later with Bouin's fixative. The brains were dissected so that the entire neostriatum, nucleus accumbens, substantia nigra, and ventral tegmental area on the right side of the brain wcre in the samc block of tissue. The blocks were embedded in paraffin and sectioned at $6 \mu \mathrm{m}$. Every 10 th section through the nucleus accumbens and neostriatum was saved. After deparaffinization in xylenes and rehydration in graded alcohols, the slides were stained with hematoxylin.

The total number of medium-sized neurons in the nucleus accumbens and neostriatum on the right side of the brain was estimated using a previously described method (Bayer, 1982; Bayer et al., 1982). Briefly, we based our calculations on the equation $N=V_{t} / V_{a}$, where $N$ is the total number of cells, $V_{t}$ is the total volume of all medium-sized neuronal nuclei, and $V_{a}$ is the average volume of an individual nucleus. Both $V_{t}$ and $V_{a}$ were estimated with statistical techniques, and the equation was solved for $N$.

The first and last sections that contained the nucleus accumbens and neostriatum and sections at approximately $60-100 \mu \mathrm{m}$ intervals in between were photographed at low magnification with a Leitz/Wild photomacroscope. Outlines of the neostriatum and nucleus accumbens were drawn onto the photographs with a rapidograph pen. One or two randomly selected areas in these structures were photographed at high magnification with a Zeiss photomicroscope III $(25 \times$ objective $)$. The areas in the high-magnification photographic prints were relocated in the microscope, and the contours around each complete and partial nucleus judged to be in a medium-sized neuron (Chang et al., 1982; Kita and Kitai, 1988) was outlined with a rapidograph pen by one of the present authors (S.A.B.). Endothelial nuclei and small dark nuclei (judged to be in oligodendroglia and microglia) were not included. Large nuclei, presumably in the cholinergic interneurons, were also not included as well as small nuclei in neurons resembling those in the islands of Calleja that were scattered through the nucleus accumbens.

The prepared photographs werc placed onto a Summagraphics digitizer interfaced to an IBM PC (PS/2, model 60). Using the low-magnification photographs, the contours of the entire neostriatum and nucleus accumbens were traced by hand with a cursor. With these data ( $\mathrm{x}$ - and $\mathrm{y}$-coordinates), the computer program determined the area of the nucleus accumbens and neostriatum in each section and calculated the total volume of each structure using the distances between sections as the z-coordinates. Using the high-magnification photographs, the outlines of the sample area, the contours of each complete nucleus, and the contours of all partial nuclei at the borders of the sample area were traced by hand with a cursor. The computer program 
used these data to determine the sample area, the individual areas of each complete and partial nuclear profile, and the proportion of each sample area that was taken up by nuclei (i.e., packing density). The total volume of each structure and the packing density of the medium-sized neuronal nuclei were used to calculate $V_{t}$. Finally, the areas of all complete nuclei were sorted into various size classes, and the average area was calculated by the iterative subtraction method of Hendry (1976). The average area was then used to determine the average volume $\left(V_{a}\right)$ of a nucleus in both the neostriatum and the nucleus accumbens.

Determining the total number of neurons in the substantia nigra and the ventral tegmental area. Using the brains of the same animals that were processed for the volumetric studies of the neostriatum and nucleus accumbens, the portion of the block that contained the substantia nigra and ventral tegmental area was cut at $6 \mu \mathrm{m}$. Every section was saved in five different sets of slides (sets A, B, C, D, and E). After deparaffinization in xylene and rehydration in graded alcohols, immunocytochemical staining for tyrosine hydroxylase was done on a Sequenza Immunoanalyzer (Shandon/Lipshaw) following previously described protocols (Triarhou et al., 1988a). One set of control slides and a corresponding set of weaver slides (e.g., set A) from all of the animals in the group were stained together so that any variations due to different staining runs were minimized. In all, four of the five sets were stained successfully, and our quantifications relied mainly on the use of the D set and some of the B set. After immunostaining, the slides were lightly counterstained with hematoxylin, dehydrated in graded alcohols, cleared in xylene, and coverslipped with Permount.

Beginning with the first section that showed a tyrosine hydroxylase-stained neuron in either the ventral tegmental area or the substantia nigra, every nuclear profile in tyrosine hydroxylase-stained cells was counted in sections approximately $30 \mu \mathrm{m}$ apart until the substantia nigra and ventral tegmental area disappeared. Nuclei in unstained cells and nuclei in stained cells that were not in the substantia nigra or ventral tegmental area were excluded. The borders of the substantia nigra and the ventral tegmental area corresponded closely to those used by German and Manaye (1993) with the following two exceptions. First, the interfascicular nucleus, a small group of neurons in the ventral midline just anterior and above the anterior interpeduncular nucleus, was included as part of the ventral tegmental area in our counts. Second, the scattered tyrosine hydroxylase-stained neurons among the fihers of the medial lemniscus were considered to be the most anterior extension of the retrorubral field, whereas German and Manaye included these neurons in the ventral tegmental area. Two of the present authors (S.A.B. and L.C.T.) conferred to standardize the divisions between the ventral tegmental area, substantia nigra, and retrorubral field on low-magnification photographs of several sections from each animal.

The actual counts were used to calculate the total number of neurons according to the equation $N_{c}=N_{r} P(t / t+d)$, where $n_{r}$ is corrected counts, $N_{a}$ is actual counts, $P$ is total number of sections/total number of sections counted, $t$ is section thickness, and $d$ is nuclear diameter (Abercrombie, 1946; Konigsmark, 1970).

To determine $d$, a series of high-magnification photographs were taken of tyrosine hydroxylase neurons in the substantia nigra and ventral tegmental area in each animal. The edge of the nucleus in each stained cell in the photographs was outlined with a rapidograph pen, and the areas of these nuclei were mea- sured with a Summagraphics digitizer interfaced to a model 60 IBM computer. A computer program calculated the nuclear diameters from the measured areas and put them into a histogram of large to small size classes. The iterative subtraction method of Hendry (1976) was used to calculate the average diameter.

Technical note: accounting for individual differences between computer operators. Because of the massive amount of data collection needed to estimate the total number of neurons in the neostriatum and nucleus accumbens (approximately 60-70 sections and 100-150 high-magnification samples for each animal), it was necessary for several different people to measure areas with the digitizer. In all, six different operators worked together to collect data. There were virtually no differences (always within $1-2 \%$ ) between operators' measurements of the large areas of the nucleus accumbens and neostriatum in the low-magnification photographic series. However, we discovered consistent differences $(20-35 \%)$ between operators' measurements of the small individual nuclear profile areas in the highmagnification photographic series. To control for these differences, each operator was tested with a randomized sequence of 10 different sets of six perfect circles in a range of sizes similar to those of the nuclei as they were magnified in the photographic prints (from $100 \mathrm{~mm}^{2}$ to $2.5 \mathrm{~mm}^{2}$ ). The computer calculated the true area of these circles using the radius, the operator's deviation from the true area, and determined a series of corrections (one for each size class of measured circles). Another computer program identified the operators who measured each sample and applied their individual correction factors to the data, using the correction factors for larger circles on larger nuclei and the correction factors for smaller circles on smaller nuclei. The result was a corrected data file that reduced the variability due to operator's individual differences.

This approach is similar to that pursued by astronomers in the first half of the 19th century. Bessel discovered that individual differences in the measurement of the transit times of stars were caused by the subjective aspects of the measurement process; this was called the personal equation (Boring, 1950). In our case, the outlining of nuclei on a digitizer is a complex psychomotor task requiring operator judgement, and each operator has a unique personal equation. Correcting the measurement error resulting from an operator's personal equation increases the reliability of the dependent variable and accordingly increases the power of the related statistical tests (Lipsey, 1990).

\section{References}

Abercrombie M (1946) Estimation of nuclear population from microtome sections. Anat Rec 94:239-247.

Bayer SA (1982) Changes in the total number of dentate granule cells in juvenile and adult rats: a correlated volumetric and $\left[{ }^{3} \mathrm{H}\right]$ thymidine autoradiographic study. Exp Brain Res 46:315-323.

Bayer SA, Altman J (1991) Neocortical development. New York: Raven.

Bayer SA, Yackel JW, Puri PS (1982) Neurons in the rat dentate gyrus granular layer substantially increase during juvenile and adult life. Science 216:890-892.

Beckstead RM, Domesick VB, Nauta WJH (1979) Efferent connections of the substantia nigra and ventral tegmental area in the rat. Brain Res 175:191-217.

Boring EG (1950) A History of experimental psychology, 2d ed. New York: Appleton-Century-Crofts.

Carlsen J, de Olmos J, Heimer L (1982) Tracing two-neuron pathways in the olfactory system by the aid of transneuronal degeneration: projections to the amygdaloid body and hippocampal formation. $\mathbf{J}$ Comp Neurol 208:196-208.

Carter S, Fibiger HC (1977) Ascending projections of presumed do- 
pamine-containing neurons in the ventral tegmentum of the rat as demonstrated by horseradish peroxidase. Neuroscience 2:569-576.

Chang HT, Wilson CJ, Kitai ST (1982) A Golgi study of rat neostriatal neurons: light microscopic analysis. J Comp Neurol 208:107-126.

Chronister RB, Sikes RW, Wood J, DeFrance JF (1980) The pattern of termination of ventral tegmental afferents into nucleus accumbens: an anterograde HRP analysis. Neurosci Lett 17:231-235.

Doucet G, Brundin P, Seth S, Murata Y, Strecker RE, Triarhou LC, Ghetti B, Björklund A (1989) Degeneration and graft-induced restoration of dopamine innervation in the weaver mouse neostriatum: a quantitative radioautographic study of $\left[{ }^{3} \mathrm{H}\right]$ dopamine uptake. Exp Brain Res 77:552-568.

Faull, RLM, Mchlcr WR (1978) The cells of origin of nigrotectal, nigrothalamic and nigrostriatal projections in the rat. Neuroscience 3:989-1002.

Fisher RS, Levine MS, Gazzara RA, Hull CD, Buchwald NA (1983) Postnatal development of caudate input neurons in the cat. J Comp Neurol 219:51-69.

Gerfen CR, Staines WA, Arbuthnott GW, Fibiger HC (1982) Crossed connections of the substantia nigra in the rat. J Comp Neurol 207: 283-303.

Gerfen CR, Herkenham M, Thibault J (1987) The neostriatal mosaic. II: Patch- and matrix-directed mesostriatal dopaminergic and nondopaminergic systems. J Neurosci 7:3915-3934.

German DC, Manaye KF (1993) Midbrain dopaminergic neurons (nuclei A8, A9, and A10): three-dimensional reconstruction in the rat. J Comp Neurol 331:297-309.

Ghetti B, Triarhou LC (1992a) Degeneration of mesencephalic dopamine neurons in weaver mutant mice. Neurochem Int [Suppl] 20: 305S-307S

Ghetti B, Triarhou LC (1992b) Combined degeneration of cerebellar granule cells and of midbrain dopamine neurons in the weaver mutant mouse. In: Progress in Parkinson's disease research (Hefti F, Weiner WJ, eds), pp 375-388. Mount Kisko, NY: Futura.

Ghetti B, Triarhou LC (1992c) Nigrostriatal aberrations induced by weaver gene are present at birth. Soc Neurosci Abstr 18:156.

Graybiel AM, Ohta K, Roffler-Tarlov S (1990) Patterns of cell and fiber vulnerability in the mesostriatal system of the mutant mouse weaver. I. Gradients and comparisons. J Neurosci 10:720-733.

Groenewegen HJ, Becker NEHM, Lohman AHM (1980) Subcortical afferents of the nucleus accumbens septi in the cat, studied with retrograde axonal transport of horseradish peroxidase and bisbenzimide. Neuroscience 5:1903-1916.

Gupta M, Felten DL, Ghetti B (1987) Selective loss of monoaminergic neurons in weaver mutant mice-an immunocytochemical study. Brain Res 402:379-382.

Hendry IA (1976) A method to correct adequately for the change in neuronal size when estimating neuronal numbers after nerve growth factor treatment. J Neurocytol 5:337-349.

Herkenham M, Moon-Edley S, Stuart J (1984) Cell clusters in the nucleus accumbens of the rat, and the mosaic relationship of opiate receptors, acetylcholinesterase and subcortical afferent terminations. Neuroscience 11:561-593.

Hökfelt T, Skirboll L, Rehfeld JF, Goldstein M, Markey K, Dann O (1980) A subpopulation of mesencephalic dopamine neurons projecting to limbic areas contains a cholecystokinin-like peptide: evidence from immunohistochemistry combined with retrograde tracing. Neuroscience 5:2093-2124.

Jimenez-Castellanos J, Graybiel AM (1987) Subdivisions of the dopamine-containing A8-A9-A10 complex identified by their differential mesostriatal innervation of striosomes and extrastriosomal matrix. Neuroscience 23:223-242.

Kalivas PW, Miller JS (1984) Neurotensin neurons in ventral tegmental area project to medial nucleus accumbens. Brain Res 300 : 157-160.

Kalsbeek A, Buijs, RM, Hofman MA, Matthijssen MAH, Pool CW, Uylings HBM (1987) Effects of neonatal thermal lesioning of the mesocortical dopaminergic projection on the development of the rat prefrontal cortex. Dev Brain Res 32:123-132.

Kaseda Y, Ghetti B, Low WC, Richter JA, Simon JR (1987) Dopamine $D_{2}$ receptors increase in dorsolateral striatum of weaver mutant mice. Brain Res 422:178-181

Kaseda Y, Ghetti B, Low WC, Norton J, Brittain H, Triarhou LC, Richter JA, Simon JR (1990) Age related changes in striatal dopamine $D_{2}$ receptor binding in weaver mice and effects of ventral mesencephalic grafts. Exp Brain Res 83:1-8.
Kita H, Kitai ST (1988) Glutamate decarboxylase immunoreactive neurons in rat neostriatum: their morphological types and populations. Brain Res 447:346-352.

Konigsmark BW (1970) Methods for the counting of neurons. In: Contemporary research methods in neuroanatomy (Nauta WJH Ebbesson SOE, eds), pp 315-340. Berlin: Springer.

Kume A, Takahashi A, Hashizume Y (1993) Neuronal cell loss of the striatonigral system in multiple system atrophy. J Neurol Sci 117:3340.

Lipsey MW (1990) Design sensitivity: statistical power for experimental research. Newbury Park: Sage.

Mensah PL (1977) The internal organization of the mouse caudate nuclcus: cvidence for cell clustcring and regional variation. Brain Res 137:53-66.

Nauta HJW, Pritz MB, Lasek RJ (1974) Afferents to the rat caudoputamen studied with horseradish peroxidase. An evaluation of a retrograde neuroanatomical research method. Brain Res 67:219-238.

Newman R. Winans SS (1980) An experimental study of the ventral striatum of the golden hamster. I. Neuronal connections of the nucleus accumbens. J Comp Neurol 191:167-192.

Ohta K, Graybiel AM, Roffler-Tarlov S (1989) Dopamine D, binding sites in the striatum of the mutant mouse weaver. Neuroscience 28 69-82.

Rakic P, Sidman RL (1973) Sequence of developmental abnormalities leading to granule cell deficit in cerebellar cortex of weaver mutant mice. J Comp Neurol 152:103-132.

Roffler-Tarlov S, Graybiel AM (1987) The postnatal development of the dopamine-containing innervation of dorsal and ventral striatum: effects of the weaver gene. J Neurosci 7:2364-2372.

Roffler-Tarlov S, Pugatch D, Graybiel AM (1990) Patterns of cell and fiber vulnerability in the mesostriatal system of the mutant mouse weaver. II. High affinity uptake sites for dopamine. J Neurosci 10: $734-740$

Rosegay H (1944) An experimental investigation of the connections between the corpus striatum and substantia nigra in the cat. J Comp Neurol 80:293-321.

Royce GJ (1978) Cells of origin of subcortical afferents to the caudate nucleus: a horseradish peroxidase study in the cat. Brain Res 153: $465-475$.

Sauer H, Oertel WH (1994) Progressive degeneration of nigrostriatal dopamine neurons following intrastriatal terminal lesions with 6-hydroxydopamine: a combined retrograde tracing and immunocytochemical study in the rat. Neuroscience 59:401-415.

Schmidt MJ, Sawyer BD, Perry KW, Fuller RW, Foremnan MM, Ghetti B (1981) Evidence for dopamine deficiency in the weaver mouse. Soc Neurosci Abstr 7:208.

Schmidt MJ, Sawyer BD, Perry KW, Fuller RW, Foreman MM, Ghetti B (1982) Dopamine deficiency in the weaver mutant mouse. J Neurosci 2:376-380

Schwab M, Agid Y, Glowinski L, Thoenen H (1977) Retrograde transport of ${ }^{125}$ I-tetanus toxin as a tool for tracing fiber connections in the central nervous system. Connections of the rostral part of the rat neostriatum. Brain Res 126:211-224.

Simon J, Richter JA, Ghetti B (1994) Age-dependent alterations in dopamine content, tyrosine hydroxylase activity and dopamine uptake in the striatum of the weaver mutant mouse. J Neurochem 62: 543-548.

Triarhou LC, Ghetti B (1987) Neuroanatomical substrate of behavioral impairment in weaver mutant mice. Exp Brain Res 68:434-435.

Triarhou LC, Low WC, Ghetti B (1986) Transplantation of ventral mesencephalic anlagen to hosts with genetic nigrostriatal dopamine deficiency. Proc Natl Acad Sci USA 83:8789-8793.

Triarhou LC, Norton J, Ghetti B (1988a) Mesencephalic dopamine cell deficit involves areas A8, A9 and A10 in weaver mutant mice. Exp Brain Res 70:256-265.

Triarhou LC, Norton J, Ghetti B (1988b) Synaptic connectivity of tyrosine hydroxylase immunoreactive nerve terminals in the striatum of normal, heterozygous and homozygous weaver mutant mice. J Neurocylol 17:221-232.

Ungerstedt U (1971) Stereotaxic mapping of the monoamine pathways in the rat brain. Acta Physiol Scand [Suppl] 367:1-29.

Veening JG, Cornelissen FM, Lieven PAJM (1980) The topical organization of the afferents to the caudoputamen of the rat. A horseradish peroxidase study. Neuroscience 5:1253-1268. 